HEALTH AND FINANCE

\title{
A first step towards transparency in pricing of medicines and scheduled substances - publication of guidelines for pharmaco-economic submissions
}

\author{
M P Stander, M Bergh, H Miller-Jansön \\ Tienie Stander, Margreet Bergh and Helen Miller-Jansön work for HEXOR Pty Ltd, South Africa, a health economics and outcomes research \\ company focused on scientific and robust health research that benefits humanity. Pharmaco-economic studies form the basis of outcomes research. It \\ is therefore relevant for HEXOR to share their views on the recently published National Guidelines on Pharmacoeconomic Submissions.
}

Corresponding author: M P Stander (tienies@hexor.co.za)

The National Department of Health of South Africa recently published guidelines for pharmaco-economic (PE) submissions in accordance with the Medicines and Related Substances Act (Act 101 of 1965), which came into effect on 1 April 2013. These guidelines relate to the compilation of PE submissions for evidence of cost-effectiveness of medicine or scheduled substances. The PE guidelines are a first step towards the creation of a mechanism whereby the value of medicine can be quantified in a transparent manner. The 'voluntary' nature of PE submissions speaks to the current lack of knowledge, understanding and capacity related to pharmaco-economics that exists in the private healthcare market. The current disconnect between the PE guidelines and the Medical Schemes Act should be addressed as a matter of urgency to provide a mechanism whereby guidance in terms of cost-effectiveness from a PE evaluation will be supported by guaranteed reimbursement by medical schemes.

S Afr Med J 2014;104(1):10-11. DOI:10.7196/SAMJ.7284

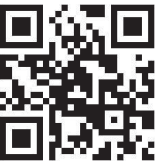

Healthcare reform in South Africa (SA) has been an ongoing process since 1994. By 2011, this process had evolved into a broad-based National Health Insurance (NHI) policy with compulsory membership for all citizens, based on a basic benefit package.

Yet another milestone was reached with the publication of the Medicines and Related Substances Act (Act 101/1965) guidelines for pharmacoeconomic (PE) submissions. ${ }^{[1]}$ The guidelines attempt to regulate and enhance a transparent pricing system for medicines and scheduled substances and offer structure for PE submissions. These guidelines came into effect on 1 April 2013.

\section{Background}

Three components 'anchor' the guidelines:

- they provide for voluntary submissions

- they apply to medicines that are registered by the Medicines Control Council in SA

- they apply to the private healthcare sector.

In the first part of the guidelines (Process for Submission), three of the stated objectives of the guidelines need to be highlighted. Firstly, there is a clear objective to move towards standardisation of PE evaluations. Secondly, there is an attempt to promote transparency when it comes to the value of medicine. The latter is important, as it is an attempt at medicine value transparency and not price transparency. The third objective is 'to create a forum which provides an objective review of the value of medicine', the key word being objective.

The Pricing Committee (PC) will be responsible for establishing the 'terms of reference for a sub-committee to assess pharmacoeconomic submissions. This is an important guideline, with far-reaching implications, as all medicines that are found to be unreasonably priced will be listed on the National Department of Health website.
This regulation specifically mentions 'unreasonably priced' and does not address 'value of medicine'.

Furthermore, the PC may only make three recommendations on the therapeutic value of submitted medicines. Firstly, it may recommend that a medicine does not offer therapeutic value relative to the single exit price (SEP). Secondly, it may recommend that it is unreasonably priced. Lastly, it may make recommendations with regard to its therapeutic value in a specific patient group or prescription by general practitioners or specialist groups, or under specific circumstances. This implies that there is no mechanism or mandate whereby the PC can make a recommendation that a medicine does in fact offer therapeutic value relative to the SEP or that it is reasonably priced.

The second part of the guidelines pertains to the content of PE evaluation submissions and addresses some technical issues that need to be complied with. It addresses critical issues such as the availability of SA clinical data v. international data, acceptable evidence (e.g. randomised controlled trials), co-administered drugs, choice of comparator drugs, and, very importantly, clinical evidence of effectiveness. The latter two require special mention as they have far-reaching implications.

With regard to the choice of a comparator medicine, the guidelines make it clear that the main comparator should be the current standard of care for local practice and go on to state '... such as those described in the Prescribed Minimum Benefits (PMB) and Essential Drugs List (EDL)'

The guidelines related to clinical outcomes (effectiveness) are comprehensive and directive and constitute the most important part of a PE submission. However, some important aspects that should be highlighted are a recommendation to include details of patient adherence to treatment data, SA quality of life measures, and transparency and completeness of search strategies for relevant data. 
One technical aspect that needs to be highlighted is the requirement to perform the PE evaluation from a third-party payer perspective. This implies that only the economic costs that are relevant to a thirdparty payer should be included in the submission. Accordingly, only direct healthcare costs should be included, with exclusion of other societal costs such as direct non-healthcare costs (transport and caretaker costs and loss of income directly associated with the disease treatment and progression) and non-direct healthcare costs (loss of productivity and the macro-economic impact on the SA economy).

The guidelines constitute 'world class' standards for PE evaluation submission and should be seen as complementary to a world-class private healthcare sector. Furthermore, they are comprehensive and directive and a significant step towards the standardisation of $\mathrm{PE}$ evaluation submissions. There is a very strong emphasis on clinical outcomes and the robustness of the supporting evidence, and to what extent the latter is transferable and applicable in SA. Mathematical modelling, when needed to mimic the natural history of the disease and estimate its economic impact by comparing a new medicine to the current practices, appears to be less important.

\section{Implications}

Several important implications need to be considered. Firstly, the PE guidelines are published as a Regulation to the Medicines and Related Substances Act (Act 101/1965). They therefore have no direct bearing on medical schemes (third-party payers in the private healthcare sector), which are regulated by the Medical Schemes Act (Act 131 of 1998, as amended). Although a manufacturer might submit a PE evaluation to the $\mathrm{PC}$ and receive a 'non-negative' outcome, this does not imply reimbursement by third-party payers. Medical schemes may or may not accept the outcome of 'good value for money' and will retain the right not to reimburse such new medicines based on arguments of affordability.

Although the Regulations clearly state that PE submissions are voluntary, this could be questioned. Paragraph 3, which deals with the criteria for medicines that require PE submissions, states three criteria and under 3(c) states: 'Where it is the opinion of the Minister, Pricing Committee or the Director-General (DG) that a pharmacoeconomic submission is necessary/required for a particular medicine.' This implies that a PE submission may become compulsory if it is so decided.

The guidelines are merely a first step towards a bigger plan in terms of transparency of the value of medicine in an NHI dispensation. This begs the question whether objectivity in a private healthcare system constitutes objectivity in a more societal system, such as NHI. Can committee members be or remain objective in an ever-changing health reform agenda, i.e. from a private healthcare focus towards an NHI-focused perspective?

The broad statement about the terms of reference that will be provided by the PC to the sub-committee ties in with the question of objectivity. In pharmacoeconomics, cost-effectiveness is based on a society's willingness-to-pay thresholds for specific benefits. It is considered an indication from society of what constitutes good value for money. On what basis will the PE sub-committee make recommendations in an objective way regarding a submission? What would their threshold for cost-effectiveness be?

Given the three recommendations that could be made by the PC, based on a PE submission, what incentives would there be for a pharmaceutical company to voluntarily submit a PE evaluation?

\section{Conclusion}

The comprehensive nature of the guidelines implies that it will be a costly project to submit a PE evaluation in line with the Regulations. These additional costs should, however, be considered against the backdrop of a shrinking private healthcare industry that is envisaged as NHI is rolled out. In this scenario, pharmaceutical companies could find it more challenging to remain profitable in SA. This will be dependent on the company's specific product portfolio and pipeline. Given this challenge, it is quite probable that some pharmaceutical companies will exit the SA market.

The publication of the PE guidelines should be considered a first step towards the creation of a mechanism whereby the value of medicine can be quantified in a transparent manner. It can be expected that voluntary submissions of PE studies will progressively move towards compulsory PE submissions. Stakeholders should therefore plan to build and enhance capacity, knowledge and insight related to pharmacoeconomics. Finally, in the future we may witness the progressive conversion of the guidelines in support of the NHI initiative and EDL extensions.

Strategically, stakeholders should not only ensure that they understand the content and implications of the PE guidelines, but also plan how they will position themselves in this changing environment.

\footnotetext{
1. Department of Health. Medicines and Related Substances Act (101/1965) Regulations relating to a transparent pricing system for medicines and scheduled substances: Publication of guidelines for rans phar ace (act) Regulations\%20relatin 4 September 2013).
}

Accepted 3 September 2013. 